\title{
Popoviciu type inequalities for $n$-convex functions via extension of Montgomery identity
}

\section{Asif R. Khan, Josip Pečarić and Marjan Praljak}

\begin{abstract}
Extension of Montgomery's identity is used in derivation of Popoviciutype inequalities containing sums $\sum_{i=1}^{m} p_{i} f\left(x_{i}\right)$, where $f$ is an $n$-convex function.

Integral analogues and some related results for $n$-convex functions at a point are also given, as well as Ostrowski-type bounds for the integral remainders of identities associated with the obtained inequalities.
\end{abstract}

\section{Introduction}

Pečarić [7] proved the following result (see also [10, p.262]):

Proposition 1. The inequality

$$
\sum_{i=1}^{m} p_{i} f\left(x_{i}\right) \geq 0
$$

holds for all convex functions $f$ if and only if the $m$-tuples $\mathbf{x}=\left(x_{1}, \ldots, x_{m}\right)$, $\mathbf{p}=\left(p_{1}, \ldots, p_{m}\right) \in \mathbb{R}^{m}$ satisfy

$$
\sum_{i=1}^{m} p_{i}=0 \quad \text { and } \quad \sum_{i=1}^{m} p_{i}\left|x_{i}-x_{k}\right| \geq 0 \text { for } k \in\{1, \ldots, m\} .
$$

Key Words: $n$-convex functions, $n$-convex functions at a point, Montgomery identity, Čebyšev functional, exponential convexity.

2010 Mathematics Subject Classification: Primary 26A51, 26D15, 26D20.

Received: 09.09.2015

Accepted: 16.02 .2016 
Since

$$
\sum_{i=1}^{m} p_{i}\left|x_{i}-x_{k}\right|=2 \sum_{i=1}^{m} p_{i}\left(x_{i}-x_{k}\right)_{+}-\sum_{i=1}^{m} p_{i}\left(x_{i}-x_{k}\right),
$$

where $y_{+}=\max (y, 0)$, it is easy to see that condition (2) is equivalent to

$\sum_{i=1}^{m} p_{i}=0, \quad \sum_{i=1}^{m} p_{i} x_{i}=0 \quad$ and $\quad \sum_{i=1}^{m} p_{i}\left(x_{i}-x_{k}\right)_{+} \geq 0$ for $k \in\{1, \ldots, m-1\}$.

Let $A$ denote the linear operator $A(f)=\sum_{i=1}^{m} p_{i} f\left(x_{i}\right)$, let $w(x, t)=(x-$ $t)_{+}$and $x_{(1)} \leq x_{(2)} \leq \ldots \leq x_{(m)}$ be the sequence $\mathbf{x}$ in ascending order. Notice that $A\left(w\left(\cdot, x_{k}\right)\right)=\sum_{i=1}^{m} p_{i}\left(x_{i}-x_{k}\right)_{+}$. For $t \in\left[x_{(k)}, x_{(k+1)}\right]$ we have

$$
A(w(\cdot, t))=A\left(w\left(\cdot, x_{(k)}\right)\right)+\left(x_{(k)}-t\right) \sum_{\left\{i: x_{i}>x_{(k)}\right\}} p_{i},
$$

so the mapping $t \mapsto A(w(\cdot, t))$ is linear on $\left[x_{(k)}, x_{(k+1)}\right]$. Furthermore, $A\left(w\left(\cdot, x_{(m)}\right)=0\right.$, so condition (3) is equivalent to

$$
\sum_{i=1}^{m} p_{i}=0, \quad \sum_{i=1}^{m} p_{i} x_{i}=0 \text { and } \sum_{i=1}^{m} p_{i}\left(x_{i}-t\right)_{+} \geq 0 \text { for every } t \in\left[x_{(1)}, x_{(m-1)}\right] .
$$

It turns out that condition (4) is appropriate for extension of Proposition 1 to the integral case and the more general class of $n$-convex functions (see e.g. [10]).

Definition 1. The $n$th order divided difference of a function $f: I \rightarrow \mathbb{R}$ at distinct points $x_{i}, x_{i+1}, \ldots, x_{i+n} \in I=[a, b] \subset \mathbb{R}$ for some $i \in \mathbb{N}$ is defined recursively by:

$$
\begin{aligned}
{\left[x_{j} ; f\right] } & =f\left(x_{j}\right), \quad j \in\{i, \ldots, i+n\} \\
{\left[x_{i}, \ldots, x_{i+n} ; f\right] } & =\frac{\left[x_{i+1}, \ldots, x_{i+n} ; f\right]-\left[x_{i}, \ldots, x_{i+n-1} ; f\right]}{x_{i+n}-x_{i}} .
\end{aligned}
$$

It may easily be verified that

$$
\left[x_{i}, \ldots, x_{i+n} ; f\right]=\sum_{k=0}^{n} \frac{f\left(x_{i+k}\right)}{\prod_{j=i, j \neq i+k}^{i+n}\left(x_{i+k}-x_{j}\right)} .
$$

Remark 1. Let us denote $\left[x_{i}, \ldots, x_{i+n} ; f\right]$ by $\Delta_{(n)} f\left(x_{i}\right)$. The value $\left[x_{i}, \ldots, x_{i+n} ; f\right]$ is independent of the order of the points $x_{i}, x_{i+1}, \ldots, x_{i+n}$. We can extend this definition by including the cases in which two or more points coincide by taking respective limits. 
Definition 2. A function $f: I \rightarrow \mathbb{R}$ is called convex of order $n$ or $n$-convex if for all choices of $(n+1)$ distinct points $x_{i}, \ldots, x_{i+n}$ we have $\Delta_{(n)} f\left(x_{i}\right) \geq 0$.

If $n$th order derivative $f^{(n)}$ exists, then $f$ is $n$-convex if and only if $f^{(n)} \geq 0$. For $1 \leq k \leq n-2$, a function $f$ is $n$-convex if and only if $f^{(k)}$ exists and is $(n-k)$-convex.

The following result is due to Popoviciu $[11,12]$ (see $[14,10,9]$ also).

Proposition 2. Let $n \geq 2$. Inequality (1) holds for all $n$-convex functions $f:[a, b] \rightarrow \mathbb{R}$ if and only if the $m$-tuples $\mathbf{x} \in[a, b]^{m}, \mathbf{p} \in \mathbb{R}^{m}$ satisfy

$$
\begin{gathered}
\sum_{i=1}^{m} p_{i} x_{i}^{k}=0, \quad \text { for all } k=0,1, \ldots, n-1 \\
\sum_{i=1}^{m} p_{i}\left(x_{i}-t\right)_{+}^{n-1} \geq 0, \quad \text { for every } t \in[a, b] .
\end{gathered}
$$

In fact, Popoviciu proved a stronger result - it is enough to assume that (6) holds for every $t \in\left[x_{(1)}, x_{(m-n+1)}\right]$ and then, due to (5), it is automatically satisfied for every $t \in[a, b]$. The integral analogue (see $[13,10,9])$ is given in the next proposition.

Proposition 3. Let $n \geq 2, p:[\alpha, \beta] \rightarrow \mathbb{R}$ and $g:[\alpha, \beta] \rightarrow[a, b]$. Then, the inequality

$$
\int_{\alpha}^{\beta} p(x) f(g(x)) d x \geq 0
$$

holds for all $n$-convex functions $f:[a, b] \rightarrow \mathbb{R}$ if and only if

$$
\begin{gathered}
\int_{\alpha}^{\beta} p(x) g(x)^{k} d x=0, \quad \text { for all } k=0,1, \ldots, n-1 \\
\int_{\alpha}^{\beta} p(x)(g(x)-t)_{+}^{n-1} d x \geq 0, \quad \text { for every } t \in[a, b] .
\end{gathered}
$$

In this paper we will prove inequalities of type (1) and (7) for $n$-convex functions by using the following extension of Montgomery's identity via Taylor's formula obtained in [1].

Proposition 4. Let $n \in \mathbb{N}, f: I \rightarrow \mathbb{R}$ be such that $f^{(n-1)}$ is absolutely continuous, $I \subset \mathbb{R}$ an open interval, $a, b \in I, a<b$. Then the following 
identity holds

$$
\begin{aligned}
f(x) & =\frac{1}{b-a} \int_{a}^{b} f(t) d t+\sum_{k=0}^{n-2} \frac{f^{(k+1)}(a)}{k !(k+2)} \frac{(x-a)^{k+2}}{b-a} \\
& -\sum_{k=0}^{n-2} \frac{f^{(k+1)}(b)}{k !(k+2)} \frac{(x-b)^{k+2}}{b-a}+\frac{1}{(n-1) !} \int_{a}^{b} T_{n}(x, s) f^{(n)}(s) d s
\end{aligned}
$$

where

$$
T_{n}(x, s)= \begin{cases}-\frac{(x-s)^{n}}{n(b-a)}+\frac{x-a}{b-a}(x-s)^{n-1}, & a \leq s \leq x, \\ -\frac{(x-s)^{n}}{n(b-a)}+\frac{x-b}{b-a}(x-s)^{n-1}, & x<s \leq b .\end{cases}
$$

In case $n=1$ the sum $\sum_{k=0}^{n-2} \ldots$ is empty, so identity (9) reduces to the well-known Montgomery identity (see for instance [6])

$$
f(x)=\frac{1}{b-a} \int_{a}^{b} f(t) d t+\int_{a}^{b} P(x, s) f^{\prime}(s) d s
$$

where $P(x, s)$ is the Peano kernel, defined by

$$
P(x, s)= \begin{cases}\frac{s-a}{b-a}, & a \leq s \leq x, \\ \frac{s-b}{b-a}, & x<s \leq b .\end{cases}
$$

The outline of the paper is as follows: in Section 2 we will use the extension of Montgomery's identity (9) to obtain inequalities of type (1) and (7) for $n$-convex functions. In Section 3 we will give related inequalities for $n$-convex functions at a point, a generalization of the class of $n$-convex functions introduced in [9]. In Section 4 we will will give bounds for the integral reminders of identities obtained in earlier sections by using Čebyšev type inequalities. In the last section we will prove certain properties of linear functionals associated with the obtained inequalities. In particular, we will construct new classes of exponentially convex functions and Cauchy type means.

\section{Popoviciu type identities and inequalities via extension of Montgomery identity}

Theorem 1. Suppose all the assumptions from Proposition 4 hold and let $T_{n}$ be given by (10). Furthermore, let $m \in \mathbb{N}, x_{i} \in[a, b]$ and $p_{i} \in \mathbb{R}$ for 
$i \in\{1,2, \ldots, m\}$ be such that $\sum_{i=1}^{m} p_{i}=0$. Then

$$
\begin{aligned}
\sum_{i=1}^{m} p_{i} f\left(x_{i}\right) & =\frac{1}{b-a}\left[\sum_{k=0}^{n-2} \frac{1}{k !(k+2)} f^{(k+1)}(a) \sum_{i=1}^{m} p_{i}\left(x_{i}-a\right)^{k+2}\right. \\
& \left.-\sum_{k=0}^{n-2} \frac{1}{k !(k+2)} f^{(k+1)}(b) \sum_{i=1}^{m} p_{i}\left(x_{i}-b\right)^{k+2}\right] \\
& +\frac{1}{(n-1) !} \int_{a}^{b}\left(\sum_{i=1}^{m} p_{i} T_{n}\left(x_{i}, s\right)\right) f^{(n)}(s) d s
\end{aligned}
$$

Proof. We take extension of Montgomery identity (9) to obtain

$$
\begin{aligned}
\sum_{i=1}^{m} p_{i} f & \left(x_{i}\right)=\frac{1}{b-a} \int_{a}^{b} f(t) d t \sum_{i=1}^{m} p_{i} \\
& +\sum_{i=1}^{m} p_{i}\left(\sum_{k=0}^{n-2} \frac{f^{(k+1)}(a)}{k !(k+2)} \frac{\left(x_{i}-a\right)^{k+2}}{b-a}-\sum_{k=0}^{n-2} \frac{f^{(k+1)}(b)}{k !(k+2)} \frac{\left(x_{i}-b\right)^{k+2}}{b-a}\right) \\
& +\frac{1}{(n-1) !} \sum_{i=1}^{m} p_{i} \int_{a}^{b} T_{n}\left(x_{i}, s\right) f^{(n)}(s) d s .
\end{aligned}
$$

By simplifying this expressions we obtain (11).

We may state its integral version as follows.

Theorem 2. Let $g:[\alpha, \beta] \rightarrow[a, b]$ and $p:[\alpha, \beta] \rightarrow \mathbb{R}$ be integrable functions such that $\int_{\alpha}^{\beta} p(x) d x=0$. Let $n \in \mathbb{N}, I \subset \mathbb{R}$ be an open interval, $a, b \in I$, $a<b, T_{n}$ be given by (10) and $f: I \rightarrow \mathbb{R}$ be such that $f^{(n-1)}$ is absolutely continuous. Then

$$
\begin{aligned}
\int_{\alpha}^{\beta} p(x) f(g(x)) d x & =\frac{1}{b-a}\left[\sum_{k=0}^{n-2} \frac{1}{k !(k+2)} f^{(k+1)}(a) \int_{\alpha}^{\beta} p(x)(g(x)-a)^{k+2} d x\right. \\
& \left.-\sum_{k=0}^{n-2} \frac{1}{k !(k+2)} f^{(k+1)}(b) \int_{\alpha}^{\beta} p(x)(g(x)-b)^{k+2} d x\right] \\
& +\frac{1}{(n-1) !} \int_{a}^{b}\left(\int_{\alpha}^{\beta} p(x) T_{n}(g(x), s) d x\right) f^{(n)}(s) . d s
\end{aligned}
$$

Proof. Our required result is obtained by using extension of Montgomery identity (9) in the following expression

$$
\int_{\alpha}^{\beta} p(x) f(g(x)) d x
$$

and then using Fubini's theorem. 
Now we state inequalities derived from the obtained identities.

Theorem 3. Let all the assumptions of Theorem 1 hold with the additional condition

$$
\sum_{i=1}^{m} p_{i} T_{n}\left(x_{i}, s\right) \geq 0, \quad \text { for all } s \in[a, b] .
$$

Then, for every $n$-convex function $f: I \rightarrow \mathbb{R}$ the following inequality holds

$$
\begin{aligned}
\sum_{i=1}^{m} p_{i} f\left(x_{i}\right) & \geq \frac{1}{b-a}\left[\sum_{k=0}^{n-2} \frac{1}{k !(k+2)} f^{(k+1)}(a) \sum_{i=1}^{m} p_{i}\left(x_{i}-a\right)^{k+2}\right. \\
& \left.-\sum_{k=0}^{n-2} \frac{1}{k !(k+2)} f^{(k+1)}(b) \sum_{i=1}^{m} p_{i}\left(x_{i}-b\right)^{k+2}\right]
\end{aligned}
$$

If the inequality in (13) is reversed, then (14) holds with the reversed sign of inequality.

Proof. The function $f$ is $n$-convex, so $f^{(n)} \geq 0$. Using this fact and (13) in (11) we easily arrive at our required result.

Remark 2. If reverse inequality holds in (13) then reverse inequality holds in (14).

Now we state an important consequence.

Theorem 4. Suppose all the assumptions from Theorem 1 hold. Additionally, let $j \in \mathbb{N}, 2 \leq j \leq n$ and let $\mathbf{x}=\left(x_{1}, \ldots, x_{m}\right) \in[a, b]^{m}, \mathbf{p}=\left(p_{1}, \ldots, p_{m}\right) \in \mathbb{R}^{m}$ satisfy (5) and (6) with $n$ replaced by $j$. If $f$ is $n$-convex and $n-j$ is even, then

$$
\begin{aligned}
\sum_{i=1}^{m} p_{i} f\left(x_{i}\right) & \geq \frac{1}{b-a}\left[\sum_{k=j-2}^{n-2} \frac{1}{k !(k+2)} f^{(k+1)}(a) \sum_{i=1}^{m} p_{i}\left(x_{i}-a\right)^{k+2}\right. \\
& \left.-\sum_{k=j-2}^{n-2} \frac{1}{k !(k+2)} f^{(k+1)}(b) \sum_{i=1}^{m} p_{i}\left(x_{i}-b\right)^{k+2}\right] .
\end{aligned}
$$

Proof. Let $s \in[a, b]$ be fixed. Notice that

$$
(b-a) T_{n}(x, s)=L_{n}(x)+(b-a)(x-s)_{+}^{n-1},
$$

where

$$
L_{n}(x)=-\frac{(x-s)^{n}}{n}+(x-b)(x-s)^{n-1}
$$


Using the Pochhammer symbol $(y)_{k}=y(y-1) \cdots(y-k+1)$ we have

$$
\begin{aligned}
L_{n}^{(j)}(x)=- & (n-1)_{j-1}(x-s)^{n-j}+\left(\begin{array}{l}
j \\
0
\end{array}\right)(x-b)(n-1)_{j}(x-s)^{n-j-1} \\
& \quad+\left(\begin{array}{l}
j \\
1
\end{array}\right)(n-1)_{j-1}(x-s)^{n-j} \\
= & (n-1)_{j-1}(x-s)^{n-j-1}[(j-1)(x-s)+(n-j)(x-b)] .
\end{aligned}
$$

Therefore, (16) and (17) for $s<x \leq b$ yield

$$
\begin{aligned}
\frac{d^{j}}{d x^{j}} & T_{n}(x, s)=\frac{1}{b-a} L_{n}^{(j)}(x)+(n-1)_{j}(x-s)^{n-j-1} \\
& =\frac{(n-1)_{j-1}}{b-a}(x-s)^{n-j-1}[(j-1)(x-s)+(n-j)(x-a)] \geq 0,
\end{aligned}
$$

while for $a \leq x<s$ we have

$$
\begin{aligned}
(-1)^{n-j} & \frac{d^{j}}{d x^{j}} T_{n}(x, s)=(-1)^{n-j} \frac{1}{b-a} L_{n}^{(j)}(x) \\
& =\frac{(n-1)_{j-1}}{b-a}(s-x)^{n-j-1}[(j-1)(s-x)+(n-j)(b-x)] \geq 0 .
\end{aligned}
$$

From (16) it is clear that $x \mapsto \frac{d^{j}}{d x^{j}} T_{n}(x, s)$ is continuous for $j \leq n-2$. Hence, if $j \leq n-2$ and $n-j$ is even, from (18) and (19) we can conclude that the function $x \mapsto T_{n}(x, s)$ is $j$-convex. Moreover, the conclusion extends to the case $j=n, i$. e. the mapping $x \mapsto T_{n}(x, s)$ is $n$-convex, since the mapping $x \mapsto \frac{d^{n-2}}{d x^{n-2}} T_{n}(x, s)$ is 2-convex.

Now, by Proposition 2, we see that assumption (13) is satisfied, so inequality (14) holds. Moreover, due to assumption (5), $\sum_{i=1}^{m} p_{i} P\left(x_{i}\right)=0$ for every polynomial $P$ of degree $\leq j-1$, so the first $j-2$ terms in the inner sum in (14) vanish, $i$. e., the right hand side of (14) under the assumptions of this theorem is equal to the right hand side of (15).

Corollary 1. Suppose all the assumptions from Theorem 1 hold. Additionally, let $j \in \mathbb{N}, 2 \leq j \leq n$, let $\mathbf{x}=\left(x_{1}, \ldots, x_{m}\right) \in[a, b]^{m}, \mathbf{p}=\left(p_{1}, \ldots, p_{m}\right) \in \mathbb{R}^{m}$ 
satisfy (5) and (6) with $n$ replaced by $j$ and denote

$$
\begin{aligned}
H(x)=\frac{1}{b-a}\left[\sum_{k=j-2}^{n-2} \frac{1}{k !(k+2)} f^{(k+1)}(a)(x-a)^{k+2}\right. & \\
& \left.-\sum_{k=j-2}^{n-2} \frac{1}{k !(k+2)} f^{(k+1)}(b)(x-b)^{k+2}\right] .
\end{aligned}
$$

If $H$ is $j$-convex on $[a, b]$ and $n-j$ is even, then

$$
\sum_{i=1}^{m} p_{i} f\left(x_{i}\right) \geq 0 \text {. }
$$

Proof. Applying Proposition 2 we conclude that the right hand side of (15) is nonnegative for the $j$-convex function $H$.

Remark 3. For example, since the functions $x \mapsto(x-a)^{k+2}$ and $x \mapsto$ $(-1)^{k-j}(x-b)^{k+2}$ are $j$-convex on $[a, b]$, the function $H$ given by (20) is $j$-convex if $f^{(k+1)}(a) \geq 0$ and $(-1)^{k+1-j} f^{(k+1)}(b) \geq 0$ for $k=j-2, \ldots, n-2$.

In the remainder of the section we will state integral versions of the previous results, the proofs of which are analogous to the discrete case.

Theorem 5. Let all the assumptions of Theorem 2 hold with the additional condition

$$
\int_{\alpha}^{\beta} p(x) T_{n}(g(x), s) d x \geq 0, \quad \text { for all } s \in[a, b] .
$$

Then, for every $n$-convex function $f: I \rightarrow \mathbb{R}$ the following inequality holds

$$
\begin{aligned}
\int_{\alpha}^{\beta} p(x) f(g(x)) d x \geq & \frac{1}{b-a}\left[\sum_{k=0}^{n-2} \frac{1}{k !(k+2)} f^{(k+1)}(a) \int_{\alpha}^{\beta} p(x)(g(x)-a)^{k+2} d x\right. \\
& \left.-\sum_{k=0}^{n-2} \frac{1}{k !(k+2)} f^{(k+1)}(b) \int_{\alpha}^{\beta} p(x)(g(x)-b)^{k+2} d x\right] .
\end{aligned}
$$

Theorem 6. Suppose all the assumptions from Theorem 2 hold. Additionally, let $j \in \mathbb{N}, 2 \leq j \leq n$ and let $p:[\alpha, \beta] \rightarrow \mathbb{R}$ and $g:[\alpha, \beta] \rightarrow[a, b]$ satisfy (8) with $n$ replaced by $j$. If $f$ is $n$-convex and $n-j$ is even, then

$$
\begin{aligned}
\int_{\alpha}^{\beta} p(x) f(g(x)) d x \geq & \frac{1}{b-a}\left[\sum_{k=j-2}^{n-2} \frac{1}{k !(k+2)} f^{(k+1)}(a) \int_{\alpha}^{\beta} p(x)(g(x)-a)^{k+2} d x\right. \\
& \left.-\sum_{k=j-2}^{n-2} \frac{1}{k !(k+2)} f^{(k+1)}(b) \int_{\alpha}^{\beta} p(x)(g(x)-b)^{k+2} d x\right] .
\end{aligned}
$$


Corollary 2. Let $j, n, f, p$ and $g$ be as in Theorem 6 and let $H$ be given by (20). If $H$ is $j$-convex and $n-j$ is even, then

$$
\int_{\alpha}^{\beta} p(x) f(g(x)) d x \geq 0 .
$$

\section{Related inequalities for $n$-convex functions at a point}

In this section we will give related results for the class of $n$-convex functions at a point introduced in [9].

Definition 3. Let $I$ be an interval in $\mathbb{R}, c$ a point in the interior of $I$ and $n \in \mathbb{N}$. A function $f: I \rightarrow \mathbb{R}$ is said to be $n$-convex at point $c$ if there exists a constant $K$ such that the function

$$
F(x)=f(x)-\frac{K}{(n-1) !} x^{n-1}
$$

is $(n-1)$-concave on $I \cap(-\infty, c]$ and $(n-1)$-convex on $I \cap[c, \infty)$. A function $f$ is said to be $n$-concave at point $c$ if the function $-f$ is $n$-convex at point $c$.

A property that explains the name of the class is the fact that a function is $n$-convex on an interval if and only if it is $n$-convex at every point of the interval (see [2, 9]). Pečarić, Praljak and Witkowski in [9] studied necessary and sufficient conditions on two linear functionals $A: C([a, c]) \rightarrow \mathbb{R}$ and $B: C([c, b]) \rightarrow \mathbb{R}$ so that the inequality $A(f) \leq B(f)$ holds for every function $f$ that is $n$-convex at $c$. In this section we will give inequalities of this type for particular linear functionals related to the inequalities obtained in the previous section.

Let $e_{i}$ denote the monomials $e_{i}(x)=x^{i}, i \in \mathbb{N}_{0}$. For the rest of this section, $A$ and $B$ will denote the linear functionals obtained as the difference of the left and right hand sides of inequality (14) applied to the intervals $[a, c]$ and $[c, b]$ respectively. More concretely, let $T_{n}^{[a, c]}$ and $T_{n}^{[c, b]}$ denote the equivalent of (10) on these intervals, i. e.,

$$
\begin{aligned}
& T_{n}^{[a, c]}(x, s)= \begin{cases}-\frac{(x-s)^{n}}{n(c-a)}+\frac{x-a}{c-a}(x-s)^{n-1}, & a \leq s \leq x, \\
-\frac{(x-s)^{n}}{n(c-a)}+\frac{x-c}{c-a}(x-s)^{n-1}, & x<s \leq c,\end{cases} \\
& T_{n}^{[c, b]}(x, s)= \begin{cases}-\frac{(x-s)^{n}}{n(b-c)}+\frac{x-c}{b-c}(x-s)^{n-1}, & c \leq s \leq x, \\
-\frac{(x-s)^{n}}{n(b-c)}+\frac{x-b}{b-c}(x-s)^{n-1}, & x<s \leq b .\end{cases}
\end{aligned}
$$


Let $\mathbf{x} \in[a, c]^{m}, \mathbf{p} \in \mathbb{R}^{m}, \mathbf{y} \in[c, b]^{l}$ and $\mathbf{q} \in \mathbb{R}^{l}$ and denote

$$
\begin{array}{r}
A(f)=\sum_{i=1}^{m} p_{i} f\left(x_{i}\right)-\frac{1}{c-a}\left[\sum_{k=0}^{n-2} \frac{1}{k !(k+2)} f^{(k+1)}(a) \sum_{i=1}^{m} p_{i}\left(x_{i}-a\right)^{k+2}\right. \\
\left.-\sum_{k=0}^{n-2} \frac{1}{k !(k+2)} f^{(k+1)}(c) \sum_{i=1}^{m} p_{i}\left(x_{i}-c\right)^{k+2}\right], \\
B(f)=\sum_{i=1}^{l} q_{i} f\left(y_{i}\right)-\frac{1}{b-c}\left[\sum_{k=0}^{n-2} \frac{1}{k !(k+2)} f^{(k+1)}(c) \sum_{i=1}^{l} q_{i}\left(y_{i}-c\right)^{k+2}\right. \\
\left.-\sum_{k=0}^{n-2} \frac{1}{k !(k+2)} f^{(k+1)}(b) \sum_{i=1}^{l} q_{i}\left(y_{i}-b\right)^{k+2}\right] .
\end{array}
$$

Notice that, using the newly introduced functionals $A$ and $B$, identity (11) applied to the intervals $[a, c]$ and $[c, b]$ can be written as

$$
\begin{aligned}
& A(f)=\frac{1}{(n-1) !} \int_{a}^{c}\left(\sum_{i=1}^{m} p_{i} T_{n}^{[a, c]}\left(x_{i}, s\right)\right) f^{(n)}(s) d s, \\
& B(f)=\frac{1}{(n-1) !} \int_{c}^{b}\left(\sum_{i=1}^{l} q_{i} T_{n}^{[c, b]}\left(y_{i}, s\right)\right) f^{(n)}(s) d s .
\end{aligned}
$$

Theorem 7. Let $\mathbf{x} \in[a, c]^{m}, \mathbf{p} \in \mathbb{R}^{m}, \mathbf{y} \in[c, b]^{l}$ and $\mathbf{q} \in \mathbb{R}^{l}$ be such that

$$
\begin{gathered}
\sum_{i=1}^{m} p_{i} T_{n}^{[a, c]}\left(x_{i}, s\right) \geq 0, \quad \text { for every } s \in[a, c] \\
\sum_{i=1}^{l} q_{i} T_{n}^{[c, b]}\left(y_{i}, s\right) \geq 0, \quad \text { for every } s \in[c, b] \\
\int_{a}^{c}\left(\sum_{i=1}^{m} p_{i} T_{n}^{[a, c]}\left(x_{i}, s\right)\right) f^{(n)}(s) d s=\int_{c}^{b}\left(\sum_{i=1}^{l} q_{i} T_{n}^{[c, b]}\left(y_{i}, s\right)\right) f^{(n)}(s) d s
\end{gathered}
$$

where $T_{n}^{[a, c]}, T_{n}^{[c, b]}, A$ and $B$ are given by (23), (24), (25) and (26) respectively. If $f:[a, b] \rightarrow \mathbb{R}$ is $(n+1)$-convex at point $c$, then

$$
A(f) \leq B(f) .
$$

If the inequalities in (29) and (30) are reversed, then (32) holds with the reversed sign of inequality. 
Proof. Let $F=f-\frac{K}{n !} e_{n}$ be as in Definition $3, i . e$. , the function $F$ is $n$-concave on $[a, c]$ and $n$-convex on $[c, b]$. Applying Theorem 3 to $F$ on the interval $[a, c]$ we have

$$
0 \geq A(F)=A(f)-\frac{K}{n !} A\left(e_{n}\right)
$$

and applying Theorem 3 to $F$ on the interval $[c, b]$ we have

$$
0 \leq B(F)=B(f)-\frac{K}{n !} B\left(e_{n}\right) .
$$

Identities (27) and (28) applied to the function $e_{n}$ yield

$$
\begin{aligned}
& A\left(e_{n}\right)=\frac{1}{(n-1) !} \int_{a}^{c}\left(\sum_{i=1}^{m} p_{i} T_{n}^{[a, c]}\left(x_{i}, s\right)\right) d s \\
& B\left(e_{n}\right)=\frac{1}{(n-1) !} \int_{c}^{b}\left(\sum_{i=1}^{l} q_{i} T_{n}^{[c, b]}\left(y_{i}, s\right)\right) d s .
\end{aligned}
$$

Therefore, assumption (31) is equivalent to $A\left(e_{n}\right)=B\left(e_{n}\right)$. Now, from (33) and (34) we obtain the stated inequality.

Remark 4. In the proof of Theorem 7 we have, actually, shown that

$$
A(f) \leq \frac{K}{n !} A\left(e_{n}\right)=\frac{K}{n !} B\left(e_{n}\right) \leq B(f) .
$$

In fact, inequality (32) still holds if we replace assumption (31) with the weaker assumption that $K\left(B\left(e_{n}\right)-A\left(e_{n}\right)\right) \geq 0$.

Corollary 3. Let $j_{1}, j_{2}, n \in \mathbb{N}, \leq j_{1}, j_{2} \leq n$, let $f:[a, b] \rightarrow \mathbb{R}$ be $(n+1)$ convex at point $c$, let $m$-tuples $\mathbf{x} \in[a, c]^{m}$ and $\mathbf{p} \in \mathbb{R}^{m}$ satisfy (5) and (6) with $n$ replaced by $j_{1}$, let $l$-tuples $\mathbf{y} \in[c, b]^{l}$ and $\mathbf{q} \in \mathbb{R}^{l}$ satisfy

$$
\begin{gathered}
\sum_{i=1}^{l} q_{i} y_{i}^{k}=0, \quad \text { for all } k=0,1, \ldots, j_{2}-1 \\
\sum_{i=1}^{l} q_{i}\left(y_{i}-t\right)_{+}^{j_{2}-1} \geq 0, \quad \text { for every } t \in\left[y_{(1)}, y_{(l-n+1)}\right]
\end{gathered}
$$

and let (31) holds. If $n-j_{1}$ and $n-j_{2}$ are even, then

$$
A(f) \leq B(f)
$$

Proof. See the proof of Theorem 4. 


\section{Bounds for identities related to the Popoviciu-type in- equalities}

Let $f, h:[a, b] \rightarrow \mathbb{R}$ be two Lebesgue integrable functions. We consider the Čebyšev functional

$$
T(f, h)=\frac{1}{b-a} \int_{a}^{b} f(x) h(x) d x-\left(\frac{1}{b-a} \int_{a}^{b} f(x) d x\right)\left(\frac{1}{b-a} \int_{a}^{b} h(x) d x\right) .
$$

The symbol $L_{p}[a, b](1 \leq p<\infty)$ denotes the space of $p$-power integrable functions on the interval $[a, b]$ equipped with the norm

$$
\|f\|_{p}=\left(\int_{a}^{b}|f(t)|^{p} d t\right)^{\frac{1}{p}}
$$

and $L_{\infty}[a, b]$ denotes the space of essentially bounded functions on $[a, b]$ with the norm

$$
\|f\|_{\infty}=\operatorname{ess} \sup _{t \in[a, b]}|f(t)| .
$$

The following results can be found in [4].

Proposition 5. Let $f:[a, b] \rightarrow \mathbb{R}$ be a Lebesgue integrable function and $h:$ $[a, b] \rightarrow \mathbb{R}$ be an absolutely continuous function with $(\cdot-a)(b-\cdot)\left[h^{\prime}\right]^{2} \in L[a, b]$. Then we have the inequality

$$
|T(f, h)| \leq \frac{1}{\sqrt{2}}\left(\frac{1}{b-a}|T(f, f)| \int_{a}^{b}(x-a)(b-x)\left[h^{\prime}(x)\right]^{2} d x\right)^{1 / 2} .
$$

The constant $\frac{1}{\sqrt{2}}$ in $(36)$ is the best possible.

Proposition 6. Let $h:[a, b] \rightarrow \mathbb{R}$ be a monotonic nondecreasing function and let $f:[a, b] \rightarrow \mathbb{R}$ be an absolutely continuous function such that $f^{\prime} \in L_{\infty}[a, b]$. Then we have the inequality

$$
|T(f, h)| \leq \frac{1}{2(b-a)}\left\|f^{\prime}\right\|_{\infty} \int_{a}^{b}(x-a)(b-x) d h(x) .
$$

The constant $\frac{1}{2}$ in $(37)$ is the best possible.

By using the aforementioned results we will obtain bounds for the integral remainders of identities obtained in Section 2. 
For $m$-tuples $\mathbf{p}=\left(p_{1}, \ldots, p_{m}\right), \mathbf{x}=\left(x_{1}, \ldots, x_{m}\right)$ with $x_{i} \in[a, b], p_{i} \in \mathbb{R}$ $(i=1, \ldots, m)$ such that $\sum_{i=0}^{m} p_{i}=0$ and the function $T_{n}$ defined as in (10), denote

$$
\delta(s)=\sum_{i=1}^{m} p_{i} T_{n}\left(x_{i}, s\right), \quad \text { for } s \in[a, b] .
$$

Similarly for functions $g:[\alpha, \beta] \rightarrow[a, b]$ and $p:[\alpha, \beta] \rightarrow \mathbb{R}$ such that $\int_{\alpha}^{\beta} p(x) d x=0$, denote

$$
\Delta(s)=\int_{\alpha}^{\beta} p(x) T_{n}(g(x), s) d x, \quad \text { for } s \in[a, b] .
$$

Now, we are ready to state the main results of this section.

Theorem 8. Let $n \in \mathbb{N}, f:[a, b] \rightarrow \mathbb{R}$ be such that $f^{(n)}$ is an absolutely continuous function with $(\cdot-a)(b-\cdot)\left[f^{(n+1)}\right]^{2} \in L[a, b], x_{i} \in[a, b]$ and $p_{i} \in \mathbb{R}$ $(i \in\{1, \ldots, m\})$ such that $\sum_{i=0}^{m} p_{i}=0$ and let the functions $T_{n}, T$ and $\delta$ be defined in (10), (35) and (38) respectively. Then

$$
\begin{aligned}
\sum_{i=1}^{m} p_{i} f\left(x_{i}\right) & =\frac{1}{b-a}\left[\sum_{k=0}^{n-2} \frac{1}{k !(k+2)} f^{(k+1)}(a) \sum_{i=1}^{m} p_{i}\left(x_{i}-a\right)^{k+2}\right. \\
& \left.-\sum_{k=0}^{n-2} \frac{1}{k !(k+2)} f^{(k+1)}(b) \sum_{i=1}^{m} p_{i}\left(x_{i}-b\right)^{k+2}\right] \\
& +\frac{\left[f^{(n-1)}(b)-f^{(n-1)}(a)\right]}{(n-1) !(b-a)} \int_{a}^{b} \delta(s) d s+R_{n}^{1}(f ; a, b),
\end{aligned}
$$

where the remainder $R_{n}^{1}(f ; a, b)$ satisfies the estimation

$$
\left|R_{n}^{1}(f ; a, b)\right| \leq \frac{1}{(n-1) !}\left(\frac{b-a}{2}\left|T(\delta, \delta) \int_{a}^{b}(s-a)(b-s)\left[f^{(n+1)}(s)\right]^{2} d s\right|\right)^{1 / 2} .
$$

Proof. If we apply Proposition 5 for $f \rightarrow \delta$ and $h \rightarrow f^{(n)}$, then we obtain

$$
\begin{gathered}
\left|\frac{1}{b-a} \int_{a}^{b} \delta(s) f^{(n)}(s) d s-\left(\frac{1}{b-a} \int_{a}^{b} \delta(s) d s\right)\left(\frac{1}{b-a} \int_{a}^{b} f^{(n)}(s) d s\right)\right| \\
\leq \frac{1}{\sqrt{2}}\left(\frac{1}{b-a}|T(\delta, \delta)| \int_{a}^{b}(s-a)(b-s)\left[f^{(n+1)}(s)\right]^{2} d s\right)^{1 / 2} .
\end{gathered}
$$


Furthermore, we have

$$
\begin{aligned}
\frac{1}{(n-1) !} \int_{a}^{b} \delta(s) f^{(n)}(s) d s & =\frac{\left[f^{(n-1)}(b)-f^{(n-1)}(a)\right]}{(n-1) !(b-a)} \int_{a}^{b} \delta(s) d s \\
& +R_{n}^{1}(f ; a, b) .
\end{aligned}
$$

where $R_{n}^{1}(f ; a, b)$ satisfies inequality (41). Now from identity (11) we obtain (40).

Here we state the integral version of previous theorem.

Theorem 9. Let $n \in \mathbb{N}, f:[a, b] \rightarrow \mathbb{R}$ be such that $f^{(n)}$ is an absolutely continuous function with $(\cdot-a)(b-\cdot)\left[f^{(n+1)}\right]^{2} \in L[a, b]$, let $g:[\alpha, \beta] \rightarrow[a, b]$ and $p:[\alpha, \beta] \rightarrow \mathbb{R}$ be functions such that $\int_{\alpha}^{\beta} p(x) d x=0$ and let the functions $T_{n}, T$ and $\Delta$ be defined in (10), (35) and (39) respectively. Then

$$
\begin{aligned}
\int_{\alpha}^{\beta} p(x) f(g(x)) d & =\frac{1}{b-a}\left[\sum_{k=0}^{n-2} \frac{1}{k !(k+2)} f^{(k+1)}(a) \int_{\alpha}^{\beta} p(x)(g(x)-a)^{k+2} d x\right. \\
& \left.-\sum_{k=0}^{n-2} \frac{1}{k !(k+2)} f^{(k+1)}(b) \int_{\alpha}^{\beta} p(x)(g(x)-b)^{k+2} d x\right] \\
+ & \frac{\left[f^{(n-1)}(b)-f^{(n-1)}(a)\right]}{(n-1) !(b-a)} \int_{a}^{b} \Delta(s) d s+R_{n}^{2}(f ; a, b),
\end{aligned}
$$

where the remainder $R_{n}^{2}(f ; a, b)$ satisfies the estimation

$\left|R_{n}^{2}(f ; a, b)\right| \leq \frac{1}{(n-1) !}\left(\frac{b-a}{2}|T(\Delta, \Delta)| \int_{a}^{b}(s-a)(b-s)\left[f^{(n+1)}(s)\right]^{2} d s\right)^{1 / 2}$.

Proof. This results easily follows by proceeding as in the proof of previous theorem and by replacing (11) by (12).

By using Proposition 6 we obtain the following Grüss type inequality.

Theorem 10. Let $n \in \mathbb{N}, f:[a, b] \rightarrow \mathbb{R}$ be such that $f^{(n)}$ is an absolutely continuous function with $f^{(n+1)} \geq 0$ on $[a, b], x_{i} \in[a, b]$ and $p_{i} \in \mathbb{R}$ $(i \in\{1, \ldots, m\})$ such that $\sum_{i=0}^{m} p_{i}=0$. Also, let the functions $T$ and $\delta$ be defined in (35) and (38) respectively. Then we have representation (40) and the remainder $R_{n}^{1}(f ; a, b)$ satisfies the following estimation

$$
\begin{aligned}
\left|R_{n}^{1}(f ; a, b)\right| \leq \frac{1}{(n-1) !}\left\|\Delta^{\prime}\right\|_{\infty}\left[\frac{b-a}{2}\left[f^{(n-1)}(b)+f^{(n-1)}(a)\right]\right. \\
\left.-\left[f^{(n-2)}(b)-f^{(n-2)}(a)\right]\right] .
\end{aligned}
$$


Proof. If we apply Proposition 6 for $f \rightarrow \delta$ and $h \rightarrow f^{(n)}$, then we obtain

$$
\begin{aligned}
\mid \frac{1}{b-a} \int_{a}^{b} \delta(s) f^{(n)}(s) d s & -\left(\frac{1}{b-a} \int_{a}^{b} \delta(s) d s\right)\left(\frac{1}{b-a} \int_{a}^{b} f^{(n)}(s) d s\right) \mid \\
& \leq \frac{1}{2(b-a)}\left\|\delta^{\prime}\right\|_{\infty} \int_{a}^{b}(s-a)(b-s) f^{(n+1)}(s) d s .
\end{aligned}
$$

Since

$$
\begin{aligned}
& \int_{a}^{b}(s-a)(b-s) f^{(n+1)}(s) d s=\int_{a}^{b}(2 s-a-b) f^{(n)}(s) d s \\
& \quad=(b-a)\left[f^{(n-1)}(b)+f^{(n-1)}(a)\right]-2\left[f^{(n-2)}(b)-f^{(n-2)}(a)\right],
\end{aligned}
$$

by using the identities (11) and (45) we deduce (44).

Next we give the integral version of the above theorem.

Theorem 11. Let $n \in \mathbb{N}, f:[a, b] \rightarrow \mathbb{R}$ be such that $f^{(n)}$ is an absolutely continuous function with $f^{(n+1)} \geq 0$ on $[a, b]$, let $g:[\alpha, \beta] \rightarrow[a, b]$ and $p:$ $[\alpha, \beta] \rightarrow \mathbb{R}$ be functions such that $\int_{\alpha}^{\beta} p(x) d x=0$. Also, let the functions $T$ and $\Delta$ be defined in (35) and (39) respectively. Then we have representation (42) and the remainder $R_{n}^{2}(f ; a, b)$ satisfies the following estimation

$$
\begin{aligned}
\left|R_{n}^{2}(f ; a, b)\right| \leq \frac{1}{(n-1) !}\left\|\Delta^{\prime}\right\|_{\infty}\left[\frac{b-a}{2}\left[f^{(n-1)}(b)+f^{(n-1)}(a)\right]\right. \\
\left.-\left[f^{(n-2)}(b)-f^{(n-2)}(a)\right]\right] .
\end{aligned}
$$

Now we state some Ostrowski-type inequalities related to the obtained identities.

Theorem 12. Let all the assumptions of Theorem 1 hold. Furthermore, let $(q, r)$ be a pair of conjugate exponents, that is $1 \leq q, r \leq \infty, \frac{1}{q}+\frac{1}{r}=1$. Let $f^{(n)} \in L_{q}[a, b]$ for some $n \in \mathbb{N}, n>1$. Then we have

$$
\begin{gathered}
\mid \sum_{i=1}^{m} p_{i} f\left(x_{i}\right)-\frac{1}{b-a}\left[\sum_{k=0}^{n-2} \frac{1}{k !(k+2)} f^{(k+1)}(a) \sum_{i=1}^{m} p_{i}\left(x_{i}-a\right)^{k+2}\right. \\
\left.-\sum_{k=0}^{n-2} \frac{1}{k !(k+2)} f^{(k+1)}(b) \sum_{i=1}^{m} p_{i}\left(x_{i}-b\right)^{k+2}\right] \mid \\
\leq \frac{1}{(n-1) !}\left\|f^{(n)}\right\|\left\|_{q}\right\| \sum_{i=1}^{m} p_{i} T_{n}\left(x_{i}, \cdot\right) \|_{r}
\end{gathered}
$$


The constant on the right hand side of (47) is sharp for $1<q \leq \infty$ and the best possible for $q=1$.

Proof. Let us denote

$$
\lambda(s)=\frac{1}{(n-1) !} \sum_{i=1}^{m} p_{i} T_{n}\left(x_{i}, s\right) .
$$

Now, by using identity (11) and applying Hölder's inequality we obtain

$$
\begin{aligned}
& \mid \sum_{i=1}^{m} p_{i} f\left(x_{i}\right)-\frac{1}{b-a}\left[\sum_{k=0}^{n-2} \frac{1}{k !(k+2)} f^{(k+1)}(a) \sum_{i=1}^{m} p_{i}\left(x_{i}-a\right)^{k+2}\right. \\
& \left.\quad-\sum_{k=0}^{n-2} \frac{1}{k !(k+2)} f^{(k+1)}(b) \sum_{i=1}^{m} p_{i}\left(x_{i}-b\right)^{k+2}\right] \mid \leq\left\|f^{(n)}\right\|_{q}\|\lambda\|_{r} .
\end{aligned}
$$

For the proof of the sharpness of the constant $\left(\int_{a}^{b}|\lambda(s)|^{r} d s\right)^{1 / r}$, let us find a function $f$ for which the equality in (48) is obtained.

For $1<q<\infty$ take $f$ to be such that

$$
f^{(n)}(s)=\operatorname{sgn} \lambda(s) \cdot|\lambda(s)|^{1 /(q-1)} .
$$

For $q=\infty$, take $f$ such that

$$
f^{(n)}(s)=\operatorname{sgn} \lambda(s) .
$$

Finally, for $q=1$, we prove that

$$
\left|\int_{a}^{b} \lambda(s) f^{(n)}(s) d s\right| \leq \max _{s \in[a, b]}|\lambda(s)| \int_{a}^{b} f^{(n)}(s) d s
$$

is the best possible inequality.

Function $T_{n}(x, \cdot)$ for $n=1$ has a jump of -1 at point $x$. But, for $n \geq 2$ it is continuous, and thus $\lambda(s)$ is continuous. Suppose that $|\lambda(s)|$ attains its maximum at $s_{0} \in[a, b]$. First we consider the case $\lambda\left(s_{0}\right)>0$. For $\epsilon$ small enough we define $f_{\epsilon}(s)$ by

$$
f_{\epsilon}(s)=\left\{\begin{aligned}
0 & , \quad a \leq s \leq s_{0} \\
\frac{1}{\epsilon n !}\left(s-s_{0}\right)^{n} & , \quad s_{0} \leq s \leq s_{0}+\epsilon, \\
\frac{1}{(n-1) !}\left(s-s_{0}\right)^{n-1} & , \quad s_{0}+\epsilon \leq s \leq b
\end{aligned}\right.
$$

We have

$$
\left|\int_{a}^{b} \lambda(s) f_{\epsilon}^{(n)}(s) d s\right|=\left|\int_{s_{0}}^{s_{0}+\epsilon} \lambda(s) \frac{1}{\epsilon} d s\right|=\frac{1}{\epsilon} \int_{s_{0}}^{s_{0}+\epsilon} \lambda(s) d s .
$$


Now, from inequality (49) we have

$$
\frac{1}{\epsilon} \int_{s_{0}}^{s_{0}+\epsilon} \lambda(s) d s \leq \lambda\left(s_{0}\right) \frac{1}{\epsilon} \int_{s_{0}}^{s_{0}+\epsilon} d s=\lambda\left(s_{0}\right) .
$$

Since

$$
\lim _{\epsilon \rightarrow 0} \frac{1}{\epsilon} \int_{s_{0}}^{s_{0}+\epsilon} \lambda(s) d s=\lambda\left(s_{0}\right),
$$

the statement follows.

In the case $\lambda\left(s_{0}\right)<0$, we define $f_{\epsilon}(s)$ by

$$
f_{\epsilon}(s)=\left\{\begin{aligned}
\frac{1}{(n-1) !}\left(s-s_{0}-\epsilon\right)^{n-1} & , \quad a \leq s \leq s_{0}, \\
-\frac{1}{\epsilon n !}\left(s-s_{0}-\epsilon\right)^{n}, & s_{0} \leq s \leq s_{0}+\epsilon, \\
0, & s_{0}+\epsilon \leq s \leq b
\end{aligned}\right.
$$

and the rest of the proof is the same as above.

Now we give the integral case of the above theorem.

Theorem 13. Let all the assumptions of Theorem 2 hold. Furthermore, let $(q, r)$ be a pair of conjugate exponents, that is $1 \leq q, r \leq \infty, \frac{1}{q}+\frac{1}{r}=1$. Let $f^{(n)} \in L_{q}[a, b]$ for some $n \in \mathbb{N}, n>1$. Then we have

$$
\begin{aligned}
& \mid \int_{\alpha}^{\beta} p(x) f(g(x)) d x \\
& -\frac{1}{b-a}\left[\sum_{k=0}^{n-2} \frac{1}{k !(k+2)} f^{(k+1)}(a) \int_{\alpha}^{\beta} p(x)(g(x)-a)^{k+2} d x\right. \\
& \left.\quad-\sum_{k=0}^{n-2} \frac{1}{k !(k+2)} f^{(k+1)}(b) \int_{\alpha}^{\beta} p(x)(g(x)-b)^{k+2} d x\right] \mid \\
& \quad \leq \frac{1}{(n-1) !}\left\|f^{(n)}\right\|_{q}\left\|\int_{\alpha}^{\beta} p(x) T_{n}(g(x), s) d x\right\|_{r} .
\end{aligned}
$$

The constant on the right hand side of (50) is sharp for $1<q \leq \infty$ and the best possible for $q=1$.

\section{Mean Value Results and Exponential Convexity}

In this section we will prove some properties of linear functionals associated with the inequalities obtained in earlier sections. Under the assumptions of 
Theorem 3 using (14) and Theorem 5 using (21) we define the following functionals respectively:

$$
\begin{aligned}
\Lambda_{1}(f)= & \sum_{i=1}^{m} p_{i} f\left(x_{i}\right)-\frac{1}{b-a}\left[\sum_{k=0}^{n-2} \frac{1}{k !(k+2)} f^{(k+1)}(a) \sum_{i=1}^{m} p_{i}\left(x_{i}-a\right)^{k+2}\right. \\
& \left.-\sum_{k=0}^{n-2} \frac{1}{k !(k+2)} f^{(k+1)}(b) \sum_{i=1}^{m} p_{i}\left(x_{i}-b\right)^{k+2}\right] \\
\Lambda_{2}(f)= & \int_{\alpha}^{\beta} p(x) f(g(x)) d x \\
- & \frac{1}{b-a}\left[\sum_{k=0}^{n-2} \frac{1}{k !(k+2)} f^{(k+1)}(a) \int_{\alpha}^{\beta} p(x)(g(x)-a)^{k+2} d x\right. \\
& \left.-\sum_{k=0}^{n-2} \frac{1}{k !(k+2)} f^{(k+1)}(b) \int_{\alpha}^{\beta} p(x)(g(x)-b)^{k+2} d x\right]
\end{aligned}
$$

Now we give mean value theorems for $\Lambda_{k}, k \in\{1,2\}$. Here $f_{0}(x)=\frac{x^{n}}{n !}$.

Theorem 14. Let $f \in C^{n}[a, b]$ and let $\Lambda_{k}: C^{n}[a, b] \rightarrow \mathbb{R}$ for $k \in\{1,2\}$ be the linear functionals as defined in $(A 1)$ and $(A 2)$ respectively. Then there exists $\xi_{k} \in[a, b]$ for $k \in\{1,2\}$ such that

$$
\Lambda_{k}(f)=f^{(n)}\left(\xi_{k}\right) \Lambda_{k}\left(f_{0}\right), \quad k \in\{1,2\}
$$

Proof. Since $f^{(n)}$ is continuous on $[a, b]$, so $L \leq f^{(n)}(x) \leq M$ for $x \in[a, b]$ where $L=\min _{x \in[a, b]} f^{(n)}(x)$ and $M=\max _{x \in[a, b]} f^{(n)}(x)$.

Therefore the function

$$
F(x)=M \frac{x^{n}}{n !}-f(x)=M f_{0}(x)-f(x)
$$

gives us

$$
F^{(n)}(x)=M-f^{(n)}(x) \geq 0
$$

i.e. $F$ is $n$-convex function. Hence $\Lambda_{k}(F) \geq 0$ and we conclude that for $k \in\{1,2\}$

$$
\Lambda_{k}(f) \leq M \Lambda_{k}\left(f_{0}\right) .
$$

Similarly, for $k \in\{1,2\}$ we have

$$
L \Lambda_{k}\left(f_{0}\right) \leq \Lambda_{k}(f) .
$$


Combining the two inequalities we get

$$
L \Lambda_{k}\left(f_{0}\right) \leq \Lambda_{k}(f) \leq M \Lambda_{k}\left(f_{0}\right)
$$

which gives us (51).

Theorem 15. Let $f, h \in C^{n}[a, b]$ and let $\Lambda_{k}: C^{n}[a, b] \rightarrow \mathbb{R}$ for $k \in\{1,2\}$ be the linear functionals as defined in $(A 1)$ and $(A 2)$ respectively. Then there exists $\xi_{k} \in[a, b]$ for $k \in\{1,2\}$ such that

$$
\frac{\Lambda_{k}(f)}{\Lambda_{k}(h)}=\frac{f^{(n)}\left(\xi_{k}\right)}{h^{(n)}\left(\xi_{k}\right)}
$$

assuming that both the denominators are non-zero.

Proof. Fix $k \in\{1,2\}$. Let $h \in C^{n}[a, b]$ be defined as

$$
\omega=\Lambda_{k}(h) f-\Lambda_{k}(f) h .
$$

Using Theorem 14 there exists $\xi_{k}$ such that

$$
0=\Lambda_{k}(\omega)=\omega^{(n)}\left(\xi_{k}\right) \Lambda_{k}\left(f_{0}\right)
$$

or

$$
\left[\Lambda_{k}(h) f^{(n)}\left(\xi_{k}\right)-\Lambda_{k}(f) h^{(n)}\left(\xi_{k}\right)\right] \Lambda_{k}\left(f_{0}\right)=0
$$

which gives us the required result.

Remark 5. If the inverse of $\frac{f^{(n)}}{h^{(n)}}$ exists, then from the above mean value theorems we can give generalized means

$$
\xi_{k}=\left(\frac{f^{(n)}}{h^{(n)}}\right)^{-1}\left(\frac{\Lambda_{k}(f)}{\Lambda_{k}(h)}\right), \quad k \in\{1,2\} .
$$

\subsection{Logarithmically Convex Functions}

A number of important inequalities arise from logarithmic convexity of some functions. In the following definitions $I$ is an interval in $\mathbb{R}$.

Definition 4. A function $f: I \rightarrow(0, \infty)$ is called log-convex in $J$-sense if the inequality

$$
f^{2}\left(\frac{x_{1}+x_{2}}{2}\right) \leq f\left(x_{1}\right) f\left(x_{2}\right)
$$

holds for each $x_{1}, x_{2} \in I$. 
Definition 5. [10, p. 7] A function $f: I \rightarrow(0, \infty)$ is called $\log$-convex if the inequality

$$
f\left(\lambda x_{1}+(1-\lambda) x_{2}\right) \leq\left[f\left(x_{1}\right)\right]^{\lambda}\left[f\left(x_{2}\right)\right]^{(1-\lambda)}
$$

holds for each $x_{1}, x_{2} \in I$ and $\lambda \in[0,1]$.

Remark 6. A function log-convex in the J-sense is log-convex if it is continuous as well.

\section{$5.2 n$-Exponentially Convex Functions}

Bernstein [3] and Widder [15] independently introduced an important sub-class of convex functions, which is called class of exponentially convex functions on a given open interval and studied some properties of this newly defined class. Pečarić and Perić in [8] introduced the notion of $n$-exponentially convex functions which is in fact generalization of the concept of exponentially convex functions. In the present subsection, we discus the same notion of $n$-exponential convexity by describing related definitions and some important results with some remarks from [8].

Definition 6. A function $f: I \rightarrow \mathbb{R}$ is $n$-exponentially convex in the $J$-sense if the inequality

$$
\sum_{i, j=1}^{n} u_{i} u_{j} f\left(\frac{t_{i}+t_{j}}{2}\right) \geq 0
$$

holds for each $t_{i} \in I$ and $u_{i} \in \mathbb{R}, i \in\{1, \ldots, n\}$.

Definition 7. A function $f: I \rightarrow \mathbb{R}$ is $n$-exponentially convex if it is $n$-exponen-tially convex in the $J$-sense and continuous on $I$.

Remark 7. We can see from the definition that 1-exponentially convex functions in the $J$-sense are in fact nonnegative functions. Also, $n$-exponentially convex functions in the $J$-sense are $k$-exponentially convex in the $J$-sense for every $k \in \mathbb{N}$ such that $k \leq n$.

Definition 8. A function $f: I \rightarrow \mathbb{R}$ is exponentially convex in the $J$-sense, if it is $n$-exponentially convex in the $J$-sense for each $n \in \mathbb{N}$.

Remark 8. A function $f: I \rightarrow \mathbb{R}$ is exponentially convex if it is $n$-exponentially convex in the $J$-sense and continuous on $I$. 
Proposition 7. If function $f: I \rightarrow \mathbb{R}$ is $n$-exponentially convex in the $J$-sense, then the matrix

$$
\left[f\left(\frac{t_{i}+t_{j}}{2}\right)\right]_{i, j=1}^{m}
$$

is positive-semidefinite. Particularly

$$
\operatorname{det}\left[f\left(\frac{t_{i}+t_{j}}{2}\right)\right]_{i, j=1}^{m} \geq 0
$$

for each $m \in \mathbb{N}, m \leq n$ and $t_{i} \in I$ for $i \in\{1, \ldots, m\}$.

Corollary 4. If function $f: I \rightarrow \mathbb{R}$ is exponentially convex, then the matrix

$$
\left[f\left(\frac{t_{i}+t_{j}}{2}\right)\right]_{i, j=1}^{m}
$$

is positive-semidefinite. Particularly

$$
\operatorname{det}\left[f\left(\frac{t_{i}+t_{j}}{2}\right)\right]_{i, j=1}^{m} \geq 0
$$

for each $m \in \mathbb{N}$ and $t_{i} \in I$ for $i \in\{1, \ldots, m\}$.

Corollary 5. If function $f: I \rightarrow(0, \infty)$ is exponentially convex, then $f$ is $\log -$

convex.

Remark 9. A function $f: I \rightarrow(0, \infty)$ is $\log$-convex in $J$-sense if and only if the inequality

$$
u_{1}^{2} f\left(t_{1}\right)+2 u_{1} u_{2} f\left(\frac{t_{1}+t_{2}}{2}\right)+u_{2}^{2} f\left(t_{2}\right) \geq 0
$$

holds for each $t_{1}, t_{2} \in I$ and $u_{1}, u_{2} \in \mathbb{R}$. It follows that a positive function is log-convex in the $J$-sense if and only if it is 2-exponentially convex in the $J$-sense. Also, using basic convexity theory it follows that a positive function is log-convex if and only if it is 2-exponentially convex.

Here, we get our results concerning the $n$-exponential convexity and exponential convexity for our functionals $\Lambda_{k}, k \in\{1,2\}$ as defined in $(A 1)$ and $(A 2)$. Throughout the section $I$ is an interval in $\mathbb{R}$. 
Theorem 16. Let $D_{1}=\left\{f_{t}: t \in I\right\}$ be a class of functions such that the function $t \mapsto\left[z_{0}, z_{1}, \ldots, z_{n} ; f_{t}\right]$ is n-exponentially convex in the $J$-sense on $I$ for any $n+1$ mutually distinct points $z_{0}, z_{1}, \ldots, z_{n} \in[a, b]$. Let $\Lambda_{k}$ be the linear functionals for $k \in\{1,2\}$ as defined in $(A 1)$ and $(A 2)$. Then the following statements are valid:

(a) The function $t \mapsto \Lambda_{k}\left(f_{t}\right)$ is $n$-exponentially convex function in the $J$-sense on $I$.

(b) If the function $t \mapsto \Lambda_{k}\left(f_{t}\right)$ is continuous on $I$, then the function $t \mapsto$ $\Lambda_{k}\left(f_{t}\right)$ is $n$-exponentially convex on $I$.

Proof. (a) Fix $k \in\{1,2\}$. Let us define the function $\omega$ for $t_{i} \in I, u_{i} \in \mathbb{R}$, $i \in\{1, \ldots, n\}$ as follows

$$
\omega=\sum_{i, j=1}^{n} u_{i} u_{j} f_{\frac{t_{i}+t_{j}}{2}}
$$

Since the function $t \mapsto\left[z_{0}, z_{1}, \ldots, z_{n} ; f_{t}\right]$ is $n$-exponentially convex in the $J$-sense, therefore

$$
\left[z_{0}, z_{1}, \ldots, z_{n} ; \omega\right]=\sum_{i, j=1}^{n} u_{i} u_{j}\left[z_{0}, z_{1}, \ldots, z_{n} ; \frac{f_{i_{i}+t_{j}}}{2}\right] \geq 0
$$

which implies that $\omega$ is $n$-convex function on $I$ and therefore $\Lambda_{k}(\omega) \geq 0$. Hence

$$
\sum_{i, j=1}^{n} u_{i} u_{j} \Lambda_{k}\left(\frac{f_{t_{i}+t_{j}}}{2}\right) \geq 0 .
$$

We conclude that the function $t \mapsto \Lambda_{k}\left(f_{t}\right)$ is an $n$-exponentially convex function on $I$ in $J$-sense.

(b) This part easily follows from definition of $n$-exponentially convex function.

As a consequence of the above theorem we give the following corollaries.

Corollary 6. Let $D_{2}=\left\{f_{t}: t \in I\right\}$ be a class of functions such that the function $t \mapsto\left[z_{0}, z_{1}, \ldots, z_{n} ; f_{t}\right]$ is an exponentially convex in the $J$-sense on I for any $n+1$ mutually distinct points $z_{0}, z_{1}, \ldots, z_{n} \in[a, b]$. Let $\Lambda_{k}$ be the linear functionals for $k \in\{1,2\}$ as defined in $(A 1)$ and $(A 2)$. Then the following statements are valid: 
(a) The function $t \mapsto \Lambda_{k}\left(f_{t}\right)$ is exponentially convex in the $J$-sense on $I$.

(b) If the function $t \mapsto \Lambda_{k}\left(f_{t}\right)$ is continuous on $I$, then the function $t \mapsto$ $\Lambda_{k}\left(f_{t}\right)$ is exponentially convex on $I$.

(c) The matrix $\left[\Lambda_{k}\left(\frac{f_{t_{i}+t_{j}}}{2}\right)\right]_{i, j=1}^{m}$ is positive-semidefinite. Particularly,

$$
\operatorname{det}\left[\Lambda_{k}\left(\frac{f_{t_{i}+t_{j}}}{2}\right)\right]_{i, j=1}^{m} \geq 0
$$

for each $m \in \mathbb{N}$ and $t_{i} \in I$ where $i \in\{1, \ldots, m\}$.

Proof. Proof follows directly from Theorem 16 by using definition of exponential convexity and Corollary 4.

Corollary 7. Let $D_{3}=\left\{f_{t}: t \in I\right\}$ be a class of functions such that the function $t \mapsto\left[z_{0}, z_{1}, \ldots, z_{n} ; f_{t}\right]$ is 2-exponentially convex in the $J$-sense on $I$ for any $n+1$ mutually distinct points $z_{0}, z_{1}, \ldots, z_{n} \in[a, b]$. Let $\Lambda_{k}$ be the linear functionals for $k \in\{1,2\}$ as defined in $(A 1)$ and $(A 2)$. Then the following statements are valid:

(a) If the function $t \mapsto \Lambda_{k}\left(f_{t}\right)$ is continuous on I, then it is 2-exponentially convex on $I$. If the function $t \mapsto \Lambda_{k}\left(f_{t}\right)$ is additionally positive, then it is also log-convex on I. Moreover, the following Lyapunov's inequality holds for $r<s<t, r, s, t \in I$

$$
\left[\Lambda_{k}\left(f_{s}\right)\right]^{t-r} \leq\left[\Lambda_{k}\left(f_{r}\right)\right]^{t-s}\left[\Lambda_{k}\left(f_{t}\right)\right]^{s-r} .
$$

(b) If the function $t \mapsto \Lambda_{k}\left(f_{t}\right)$ is positive and differentiable on $I$, then for every $s, t, u, v \in I$ such that $s \leq u$ and $t \leq v$, we have

$$
\mu_{s, t}\left(\Lambda_{k}, D_{3}\right) \leq \mu_{u, v}\left(\Lambda_{k}, D_{3}\right)
$$

where $\mu_{s, t}$ is defined as

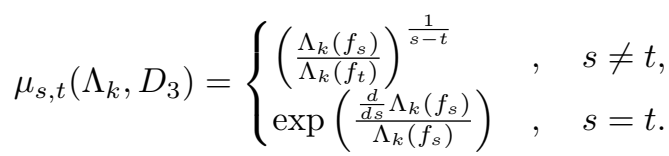

for $f_{s}, f_{t} \in D_{3}$.

Proof. (a) It follows directly form Theorem 16 and Remark 9. As the function $t \mapsto \Lambda_{k}\left(f_{t}\right)$ is log-convex, i.e., $\ln \Lambda_{k}\left(f_{t}\right)$ is convex we have

$$
\ln \left[\Lambda_{k}\left(f_{s}\right)\right]^{t-r} \leq \ln \left[\Lambda_{k}\left(f_{r}\right)\right]^{t-s}+\ln \left[\Lambda_{k}\left(f_{t}\right)\right]^{s-r}, \quad k \in\{1,2\}
$$

which gives us (53). 
(b) For a convex function $f$, the inequality

$$
\frac{f(s)-f(t)}{s-t} \leq \frac{f(u)-f(v)}{u-v}
$$

holds for all $s, t, u, v \in I \subset \mathbb{R}$ such that $s \leq u, t \leq v, s \neq t, u \neq v$.

Since $\Lambda\left(f_{t}\right)$ is $\log$-convex by $(c)$, setting $f(t)=\ln \Lambda\left(f_{t}\right)$ in (56) we have

$$
\frac{\ln \Lambda_{k}\left(f_{s}\right)-\ln \Lambda_{k}\left(f_{t}\right)}{s-t} \leq \frac{\ln \Lambda_{k}\left(f_{u}\right)-\ln \Lambda_{k}\left(f_{v}\right)}{u-v}
$$

for $s \leq u, t \leq v, s \neq t, u \neq v$, which is equivalent to (55). The cases for $s=t$ and/or $u=v$ are easily derived from (57) by taking respective limits.

Remark 10. The results from Theorem 16 and Corollaries 6 and 7 still hold when any two (all) points $z_{0}, z_{1}, \ldots, z_{n} \in[a, b]$ coincide for a family of differentiable ( $n$-times differentiable) functions $f_{t}$ such that the function $t \mapsto\left[z_{0}, z_{1}, \ldots, z_{n} ; f_{t}\right]$ is $n$-exponentially convex, exponentially convex and 2-expoenetially convex in the $J$-sense respectively.

Now, we give two important remarks and one useful corollary from [5], which we will use in some examples in the next section.

Remark 11. We will say that $\mu_{s, t}\left(\Lambda_{k}, \Omega\right)$ defined with (55) is a mean if

$$
a \leq \mu_{s, t}\left(\Lambda_{k}, \Omega\right) \leq b
$$

for $s, t \in I$ and $k \in\{1,2\}$, where $\Omega=\left\{f_{t}: t \in I\right\}$ is a family of functions and $[a, b] \subset \operatorname{Dom}\left(f_{t}\right)$.

Theorems 16 give us the following corollary.

Corollary 8. Let $a, b \in \mathbb{R}$ and $\Lambda_{k}$ be linear functionals for $k \in\{1,2\}$. Let $\Omega=\left\{f_{t}: t \in I\right\}$ be a family of functions in $C^{2}[a, b]$. If

$$
a \leq\left(\frac{\frac{d^{2} f_{s}}{d x^{2}}}{\frac{d^{2} f_{t}}{d x^{2}}}\right)^{\frac{1}{s-t}}(\xi) \leq b,
$$

for $\xi \in[a, b], s, t \in I$, then $\mu_{s, t}\left(\Lambda_{k}, \Omega\right)$ is a mean for $k \in\{1,2\}$.

Remark 12. In some examples, we will get means of this type:

$$
\left(\frac{\frac{d^{2} f_{s}}{d x^{2}}}{\frac{d^{2} f_{t}}{d x^{2}}}\right)^{\frac{1}{s-t}}(\xi)=\xi, \quad \xi \in[a, b], \quad s \neq t .
$$




\section{Examples with Applications}

In this section, we use various classes of functions $\Omega=\left\{f_{t}: t \in I\right\}$, where $I$ is an interval in $\mathbb{R}$, to construct different examples of exponentially convex functions and Stolarsky-type means. Let us consider some examples.

Example 1. Let $\Omega_{1}=\left\{\psi_{t}: \mathbb{R} \rightarrow[0, \infty): t \in \mathbb{R}\right\}$ be a family of functions defined by

$$
\psi_{t}(x)=\left\{\begin{array}{lll}
\frac{e^{t x}}{t^{n}} & , \quad t \neq 0, \\
\frac{x^{n}}{n !}, & t=0 .
\end{array}\right.
$$

Since $\frac{d^{n}}{d x^{n}} \psi_{t}(x)=e^{t x}>0$, the function $\psi_{t}(x)$ is a $n$-convex on $\mathbb{R}$ for every $t \in \mathbb{R}$ and $t \rightarrow \frac{d^{n}}{d x^{n}} \psi_{t}(x)$ is exponentially convex by definition. Using analogous arguing as in the proof of Theorems 16 , we have that $t \mapsto\left[z_{0}, z_{1}, \ldots, z_{n} ; \psi_{t}\right]$ is exponentially convex (and so exponentially convex in the J-sense). Using Corollary 6 we conclude that $t \mapsto \Lambda_{k}\left(\psi_{t}\right), k \in\{1,2\}$ are exponentially convex in the $J$-sense. It is easy to see that these mappings are continuous, so they are exponentially convex.

Assume that $t \mapsto \Lambda_{k}\left(\psi_{t}\right)>0$ for $k \in\{1,2\}$. By introducing convex functions $\psi_{t}$ in (52), we obtain the following means: for $k \in\{1,2\}$

$$
\mathfrak{M}_{s, t}\left(\Lambda_{k}, \Omega_{1}\right)= \begin{cases}\frac{1}{s-t} \ln \left(\frac{\Lambda_{k}\left(\psi_{s}\right)}{\Lambda_{k}\left(\psi_{t}\right)}\right) & , \quad s \neq t, \\ \frac{\Lambda_{k}\left(i d \cdot \psi_{s}\right)}{\Lambda_{k}\left(\psi_{s}\right)}-\frac{n}{s} & , \quad s=t \neq 0, \\ \frac{\Lambda_{k}\left(i d \cdot \psi_{0}\right)}{(n+1) \Lambda_{k}\left(\psi_{0}\right)} & , \quad s=t=0 .\end{cases}
$$

where id stands for identity function on $\mathbb{R}$. Here $\mathfrak{M}_{s, t}\left(\Lambda_{k}, \Omega_{1}\right)=\ln \left(\mu_{s, t}\left(\Lambda_{k}, \Omega_{1}\right)\right)$, $k \in\{1,2\}$ are in fact means.

Remark 13. We observe here that $\left(\frac{\frac{d^{n} \psi_{s}}{d^{n} \psi_{t}}}{\frac{d^{n}}{x^{n}}}\right)^{\frac{1}{s-t}}(\ln \xi)=\xi$ is a mean for $\xi \in[a, b]$ where $a, b \in \mathbb{R}_{+}$.

Example 2. Let $\Omega_{2}=\left\{\varphi_{t}:(0, \infty) \rightarrow \mathbb{R}: t \in \mathbb{R}\right\}$ be a family of functions defined as

$$
\varphi_{t}(x)=\left\{\begin{array}{cl}
\frac{(x)^{t}}{\frac{(t-1) \cdots(t-n+1)}{t(x)^{j} \ln (x)}} \quad, \quad t \notin\{0, \ldots, n-1\}, \\
\frac{(-1)^{n-1-j} j !(n-1-j) !}{(x)}, t=j \in\{0, \ldots, n-1\} .
\end{array}\right.
$$

Since $\varphi_{t}(x)$ is $n$-convex function for $x \in(0, \infty)$ and $t \mapsto \frac{d^{2}}{d x^{2}} \varphi_{t}(x)$ is exponentially convex, so by the same arguments given in previous example we conclude that $\Lambda_{k}\left(\varphi_{t}\right), k \in\{1,2\}$ are exponentially convex. 
We assume that $\Lambda_{k}\left(\varphi_{t}\right)>0$ for $k \in\{1,2\}$. For this family of convex functions we obtain the following means: for $k \in\{1,2\}, J=\{0,1, \ldots, n-1\}$

$\mathfrak{M}_{s, t}\left(\Lambda_{k}, \Omega_{2}\right)= \begin{cases}\left(\frac{\Lambda_{k}\left(\varphi_{s}\right)}{\Lambda_{k}\left(\varphi_{t}\right)}\right)^{\frac{1}{s-t}} & , s \neq t, \\ \exp \left((-1)^{n-1}(n-1) ! \frac{\Lambda_{k}\left(\varphi_{0} \varphi_{s}\right)}{\Lambda_{k}\left(\varphi_{s}\right)}+\sum_{k=0}^{n-1} \frac{1}{k-t}\right) & , s=t \notin J \\ \exp \left((-1)^{n-1}(n-1) ! \frac{\Lambda_{k}\left(\varphi_{0} \varphi_{s}\right)}{2 \Lambda_{k}\left(\varphi_{s}\right)}+\sum_{k=0, k \neq t}^{n-1} \frac{1}{k-t}\right) & , s=t \in J .\end{cases}$

Here $\mathfrak{M}_{s, t}\left(\Lambda_{k}, \Omega_{2}\right)=\mu_{s, t}\left(\Lambda_{k}, \Omega_{2}\right), \quad k \in\{1,2\}$ are in fact means.

Remark 14. Further, in this choice of family $\Omega_{2}$, we have

$$
\left(\frac{\frac{d^{n} \varphi_{s}}{d x^{n}}}{\frac{d^{n} \varphi_{t}}{d x^{n}}}\right)^{\frac{1}{s-t}}(\xi)=\xi, \quad \xi \in[a, b], s \neq t, \text { where } a, b \in(0, \infty) .
$$

So, using Remark 12 we have an important conclusion that $\mu_{s, t}\left(\Lambda_{k}, \Omega_{2}\right)$ is in fact mean for $k \in\{1,2\}$.

Example 3. Let $\Omega_{3}=\left\{\theta_{t}:(0, \infty) \rightarrow(0, \infty): t \in(0, \infty)\right\}$ be a family of functions defined by

$$
\theta_{t}(x)=\frac{e^{-x \sqrt{t}}}{t^{n / 2}}
$$

Since $t \mapsto \frac{d^{n}}{d x^{n}} \theta_{t}(x)=e^{-x \sqrt{t}}$ is exponentially convex for $x>0$, being the Laplace transform of a nonnegative function [5]. So, by same argument given in Example 1 we conclude that $\Lambda_{k}\left(\theta_{t}\right), k \in\{1,2\}$ are exponentially convex.

We assume that $\Lambda_{k}\left(\theta_{t}\right)>0$ for $k \in\{1,2\}$. For this family of functions we have the following possible cases of $\mu_{s, t}\left(\Lambda_{k}, \Omega_{3}\right)$ : for $k \in\{1,2\}$

$$
\mathfrak{M}_{s, t}\left(\Lambda_{k}, \Omega_{3}\right)= \begin{cases}\left(\frac{\Lambda_{k}\left(\theta_{s}\right)}{\Lambda_{k}\left(\theta_{t}\right)}\right)^{\frac{1}{s-t}} & , \quad s \neq t \\ \exp \left(-\frac{\Lambda_{k}\left(i d . \theta_{s}\right)}{2 \sqrt{s} \Lambda_{k}\left(\theta_{s}\right)}-\frac{n}{2 s}\right) & , \quad s=t\end{cases}
$$

By $(52), \mathfrak{M}_{s, t}\left(\Lambda_{k}, \Omega_{3}\right)=-(\sqrt{ } s+\sqrt{ } t) \ln \mu_{s, t}\left(\Lambda_{k}, \Omega_{3}\right), \quad k \in\{1,2\}$ defines $a$ class of means.

Example 4. Let $\Omega_{4}=\left\{\phi_{t}:(0, \infty) \rightarrow(0, \infty): t \in(0, \infty)\right\}$ be a family of functions defined by

$$
\phi_{t}(x)=\left\{\begin{array}{lll}
\frac{t^{-x}}{(\ln t)^{n}} & , & t \neq 1 \\
\frac{x^{n}}{n} & , & t=1
\end{array}\right.
$$


Since $\frac{d^{n}}{d x^{n}} \phi_{t}(x)=t^{-x}=e^{-x \ln t}>0$ for $x>0$, so by same argument given in Example 1 we conclude that $t \mapsto \Lambda_{k}\left(\phi_{t}\right), k \in\{1,2\}$ are exponentially convex.

We assume that $\Lambda_{k}\left(\phi_{t}\right)>0$ for $k \in\{1,2\}$. For this family of functions we have the following possible cases of $\mu_{s, t}\left(\Lambda_{k}, \Omega_{4}\right)$ : for $k \in\{1,2\}$

$$
\mathfrak{M}_{s, t}\left(\Lambda_{k}, \Omega_{4}\right)= \begin{cases}\left(\frac{\Lambda_{k}\left(\phi_{s}\right)}{\Lambda_{k}\left(\phi_{t}\right)}\right)^{\frac{1}{s-t}} & , \quad s \neq t, \\ \exp \left(-\frac{\Lambda_{k}\left(i d . \phi_{s}\right)}{s \Lambda_{k}\left(\phi_{s}\right)}-\frac{n}{s \ln s}\right), & s=t \neq 1, \\ \exp \left(-\frac{1}{(n+1)} \frac{\Lambda_{k}\left(i d . \phi_{1}\right)}{\Lambda_{k}\left(\phi_{1}\right)}\right) & , \quad s=t=1 .\end{cases}
$$

By $(52), \mathfrak{M}_{s, t}\left(\Lambda_{k}, \Omega_{4}\right)=-L(s, t) \ln \mu_{s, t},\left(\Lambda_{k}, \Omega_{4}\right), \quad k \in\{1,2\}$ defines a class of means, where $L(s, t)$ is Logarithmic mean defined as:

$$
L(s, t)=\left\{\begin{array}{rc}
\frac{s-t}{\ln s-\ln t}, & s \neq t, \\
s & , \quad s=t .
\end{array}\right.
$$

Remark 15. Monotonicity of $\mu_{s, t}\left(\Lambda_{k}, \Omega_{j}\right)$ follow form (54) for $k \in\{1,2\}$, $j \in\{1,2,3,4\}$.

\section{References}

[1] A. Aglić Aljinović, J. Pečarić, and A. Vukelić, On some Ostrowski type inequalities via Montgomery identity and Taylor's formula II, Tamkang Jour. Math. 36 (4), (2005), 279-301.

[2] I. A. BAloch, J. PeČArić And M. Praljak, Generalization of Levinson's inequality, J. Math. Inequal. 9 (2015), 571-586.

[3] S. N. Bernstein, Sur les fonctions absolument monotones, Acta Math. 52 (1) (1929), 1-66.

[4] P. Cerone and S. S. Dragomir, Some new Owstrowski-type bounds for the Čebyšev functional and applications, J. Math. Inequal., 8(1) (2014), 159-170.

[5] J.Jakšetić and J. Pečarić, Exponential convexity method, J. Convex Anal., 20 (1) (2013), 181-197.

[6] D. S. Mitrinović, J. E. Pečarić, and A. M. Fink, Inequalities for functions and their Integrals and Derivatives, Kluwer Academic Publishers, Dordrecht, 1994.

[7] J. Pečarić, On Jessens Inequality for Convex Functions, III, J. Math. Anal. Appl., 156 (1991), 231-239. 
[8] J. Pečarić and J. Perić, Improvements of the Giaccardi and the Petrović inequality and related Stolarsky type means, An. Univ. Craiova Ser. Mat. Inform., 39 (1) (2012), 65-75.

[9] J. Pečarić, M. Praljak and A. Witkowski, Linear operator inequality for n-convex functions at a point, Math. Ineq. Appl. 18 (2015), 1201-1217.

[10] J. E. Pečarić, F. Proschan and Y. L. Tong, Convex functions, partial orderings and statistical applications, Academic Press, New York, 1992.

[11] T. Popoviciu, Notes sur les fonctions convexes d'orde superieur III, Mathematica (Cluj) 16, (1940), 74-86.

[12] T. Popoviciu, Notes sur les fonctions convexes d'orde superieur IV, Disqusitiones Math. 1, (1940), 163-171.

[13] T. Popoviciu, Notes sur les fonctions convexes d'orde superieur IX, Bull. Math. Soc. Roumaine Sci. 43, (1941), 85-141.

[14] T. Popoviciu, Les fonctions convexes, Herman and Cie, Editeurs, Paris 1944.

[15] D. V. Widder, Necessary and sufficient conditions for the representation of a function by a doubly infinite Laplace integral, Bull. Amer. Math. Soc., 40 (4) (1934), 321-326.

Asif R. KHAN,

Department of Mathematical Sciences,

Faculty of Science, University of Karachi,

University Road, Karachi-75270, Pakistan.

Email: asifrk@uok.edu.pk

Josip PEČARIĆ,

Faculty of Textile Technology,

University of Zagreb,

Prilaz baruna Filipovića 28A, 10000 Zagreb, Croatia.

Email: pecaric@hazu.hr

Marjan PRALJAK,

Faculty of Food Technology and Biotechnology,

University of Zagreb,

Pierottijeva 6, Zagreb, Croatia.

Email: mpraljak@pbf.hr 